\title{
Nrf2 Deficiency in Dendritic Cells Enhances the Adjuvant Effect of Ambient Ultrafine Particles on Allergic Sensitization
}

\author{
Ning $\mathrm{Li}^{\mathrm{a}}{ }^{\mathrm{b}}$ Meiying Wang ${ }^{\mathrm{b}}$ Berenice Barajas $^{\mathrm{b}}$ Constantinos Sioutas $^{c}$ \\ Marc A. Williams ${ }^{d}$ Andre E. Nel ${ }^{b}$ \\ a Department of Pathology and Diagnostic Investigation, Michigan State University, East Lansing, Mich., \\ ${ }^{b}$ Division of NanoMedicine, Department of Medicine, University of California Los Angeles, and 'Department of Civil \\ and Environmental Engineering, University of Southern California, Los Angeles, Calif., and d Cardiopulmonary and \\ Immunotoxicology Branch, NHEERL, US Environmental Protection Agency, Research Triangle Park, N.C., USA
}

\section{Key Words}

Nuclear factor (erythroid-derived 2)-like 2 - Dendritic cell . Adjuvant effect - Allergic sensitization - Lung inflammation . Ultrafine particles · Interleukin-12p70 • Interleukin-6 .

Thelper 2

\section{Abstract}

Particulate matter (PM) is an important risk factor for asthma. Generation of oxidative stress by PM is a major mechanism of its health effects. Transcription factor nuclear factor (erythroid-derived 2)-like 2 (Nrf2) mediates antioxidant and phase Il enzymes and is essential in protecting against oxidative stress and lung inflammation. We have previously shown that ambient ultrafine particles (UFP) could exert a potent adjuvant effect on allergic sensitization to ovalbumin (OVA) in mice. We hypothesized that Nrf2 deficiency in dendritic cells (DC) could enhance the adjuvant potential of UFP on allergic sensitization. We show that the adjuvant effect of intranasally instilled UFP is significantly enhanced in Nrf2 knockout $\left(\mathrm{Nrf2}^{--}\right)$mice compared with their wild-type $\left(\mathrm{Nrf2}^{+/+}\right)$counterparts. Under resting conditions, $\mathrm{Nrf2}^{-/-} \mathrm{DC}$ displayed an intrinsic predilection to a T helper 2-favoring cytokine profile characterized by a low level of IL-12p70 and a high level of IL-6 as compared to $\mathrm{Nrf2}^{+/+}$DC. Adoptive transfer of OVA/UFP-treated Nrf2 ${ }^{-/-}$DC provoked a more severe allergic inflammation in the lung than $\mathrm{Nrf}^{+/+} \mathrm{DC}$ in the same treatment group. We conclude that Nrf2 deficiency in DC may promote a constitutive immune-polarizing cytokine milieu, which we propose may have contributed to the augmented adjuvant effect of UFP on allergic sensitization.

Copyright $\odot 2013$ S. Karger AG, Basel

\section{Introduction}

Exposure to airborne particulate matter (PM) is a major risk factor for the exacerbation of several existing airway diseases including asthma and allergic rhinitis $[1,2]$. One of the major sources of ambient PM is combustionderived vehicular emissions such as diesel exhaust particles (DEP) and ambient ultrafine particles (UFP). Trafficrelated PM poses a particular health risk in part because of the high levels of organic chemicals such as polycyclic aromatic hydrocarbons (PAH) and quinones as well as heavy metals and transition elements. The generation of reactive oxygen species by these chemicals or inorganic metals/elements and the subsequent induction of cellular oxidative stress has been identified as one of the key mechanistic pathways by which PM exerts its adverse

\section{KARGER}

E-Mail karger@karger.com

www.karger.com/jin
(C) 2013 S. Karger AG, Basel

$1662-811 X / 13 / 0056-0543 \$ 38.00 / 0$ 
health effects including exacerbation of preexisting allergic airway inflammation such as asthma $[2,3]$.

A crucial pathway that protects cells from oxidant injury is the antioxidant and phase II enzyme defense system mediated by the transcription factor nuclear factor (erythroid-derived 2)-like 2 (Nrf2) [3-6]. Under normal physiological conditions, Nrf2 is sequestered in the cytoplasm by its inhibitor Keap-1 and constantly targeted for proteasomal degradation. Under conditions of oxidative stress, Keap-1 releases Nrf2 from proteasomal degradation and thus allows Nrf2 to translocate to the nucleus where it is capable of activating more than 200 antioxidant and phase II enzyme genes [2, 4]. Increasing evidence has demonstrated that cellular exposure to prooxidative airborne particulates such as DEP and ambient UFP could lead to the activation of Nrf2 and subsequent induction of several antioxidant and phase II enzymes $[7,8]$. Moreover, studies have shown that in addition to protecting cells against oxidative and electrophilic injury, Nrf2 signaling is also involved in attenuating inflammation-mediated respiratory disorders such as asthma and emphysema [5]. During the early-phase of inflammation-mediated tissue damage, activation of Nrf2ARE may dampen the production or expression of inflammatory mediators including cytokines, chemokines and cell adhesion molecules. The 'anti-inflammatory' function of Nrf2 is likely the result of a cooperation of Nrf2-targeted genes in regulating the innate immune response, leading to the suppression of proinflammatory genes $[2,5]$. Thus, deficiency or complete loss of Nrf2 signaling may enhance the susceptibility not only to oxidative and electrophilic stress but also to inflammatory tissue injury including those induced by environmental pollutants.

$\mathrm{Nrf} 2$ is also required for preventing and resolving oxidant-induced acute inflammation in the lung and Nrf2 deficiency in the airway epithelium alone is sufficient to promote inflammation [9]. Deficiency of Nrf2 may enhance susceptibility to the proallergic and proinflammatory effects of particulate air pollutants, and this is supported by in vivo studies using an ovalbumin (OVA) sensitization model. In this system, repeated inhalation exposure of Nrf2 knockout $\left(\mathrm{Nrf}^{-/-}\right)$mice to low-dose DEP over an 8-week period promoted a more severe allergic inflammation in the lung and augmented airway hyperresponsiveness as compared to their wild-type Nrf2 $\left(\mathrm{Nrf}_{2}^{+/+}\right)$counterparts $[10,11]$.

Although the exact mechanism for the protective effect of Nrf2 against allergic airway inflammation remains unclear, accumulating evidence suggests that it is medi- ated by the ability of Nrf2 to regulate both cellular redox status and the balance between T helper 1 (Th1) and T helper 2 (Th2) immune responses in the lung [12]. One possible mechanism by which Nrf2 deficiency interferes with immune response is by disturbing the redox homeostasis of dendritic cells (DC), leading to an alteration in DC function [13-16]. This data contributes to our understanding of the proposed adjuvant effect of particulate pollutants including DEP and ambient PM in allergic airway inflammatory diseases such as asthma [16]. That DC are targets of PM has been reported by a number of studies. Acute exposure to ambient PM alone was shown to be capable of activating lung DC, which when cultured ex vivo promoted a Th2 cytokine response by naïve $\mathrm{CD} 4+\mathrm{T}$ cells [17]. It has also been found that PM species such as DEP and urban aerosols could enhance the expression of costimulatory molecules (CD40, CD80 and CD86) and MHC class II on the surface of DC, increase the production of proinflammatory and Th2 proallergic cytokines and stimulate naïve CD4+ $\mathrm{T}$ cell proliferation in allogeneic assays [17]. Disruption of Nrf2 in DC could promote PM-induced cellular oxidative stress, augment the cell surface expression of costimulatory molecules on DC and skew DC-mediated immune response towards a proallergic Th2-mediated pattern of immunity [16].

In this study, we tested the hypothesis that absence of Nrf2 in DC could enhance the adjuvant effect of ambient UFP on allergic sensitization. We report that Nrf2 played an important role in mediating the adjuvant effect of UFP and this occurred at the level of functional DC.

\section{Materials and Methods}

\section{Animals}

Eight- to 10-week-old female BALB/c mice were obtained from the Jackson Laboratory. These mice were used for intranasal sensitization studies and as recipients for adoptive transfer experiments. $\mathrm{Nrf} 2^{+/+}$and $\mathrm{Nrf}^{-/-}$mice on C57BL/6 background were previously bred in our laboratory and backcrossed onto BALB/c background for 10 generations [18]. The Chancellor's Animal Research Committee at UCLA approved animal housing conditions, breeding, and all the experiments described in this report.

\section{Reagents}

The limulus amebocyte lysate assay kit for endotoxin detection and endotoxin removing gel columns were obtained from Lonza (Walkerville, Md., USA) and Pierce (Rockford, Ill., USA), respectively. OVA grade V and dithiothreitol (DTT) were obtained from Sigma-Aldrich (St. Louis, Mo., USA). Ketamine and xylazine were purchased from Phoenix Pharmaceutical Inc. (St. Joseph, Mo., USA). RNeasy mini kit and RNase-Free DNase set were purchased from Qiagen (Valencia, Calif., USA). PCR reagents for Nrf2 geno- 
typing were obtained from Perkin Elmer (San Jose, Calif., USA). RPMI1640 medium and antibiotic/antimycotic mixtures were purchased from Invitrogen (Carlsbad, Calif., USA). Fetal bovine serum (FBS) was obtained from Gemini (West Sacramento, Calif., USA). Both mouse GM-CSF and IL-4 were from R\&D Systems (Minneapolis, Minn., USA). Anti-CD11c-PE, anti-MHCII-FITC, anti-CD80-FITC, anti-CD86-FITC and anti-CD40-FITC were obtained from BD Pharmingen (San Diego, Calif., USA). ELISA kits for IL-12p70, IL-6, IL- $1 \beta$ and IL-13 were purchased from BD Biosciences (San Diego, Calif., USA).

\section{Nrf2 Genotyping by PCR}

Nrf2 genotyping was performed by PCR using an aliquot of 0.5 $\mu \mathrm{l}$ of the DNA sample in a master mix containing $\mathrm{MgCl}_{2}(40 \mathrm{mM})$, dNTP $(10 \mathrm{~mm} /$ each $)$ and primers mix $(5 \mathrm{pM})$ in an AmpliTaq buffer from Perkin-Elmer. The primer mix contained 3 primers, which include a common $5^{\prime}$ primer in intron-2 (GCCTGAGAGCTGTAGGCCC), a 3 ' primer in exon-3 specific to the wild-type $\left(\mathrm{Nrf}_{2}^{+/+}\right)$allele (GGAATGGAAAATAGCTCCTGCC) and the M13-40 primer specific to the knockout $\left(\mathrm{Nrf}^{-/-}\right)$allele (GGGTTTTCCCAGTCACGAC) [18]. PCR was carried out in a robocycler with a heated top from Stratagene with 1 cycle at $94^{\circ} \mathrm{C} 1 \mathrm{~min}$ $30 \mathrm{~s}, 35 \mathrm{cycles}$ at $94^{\circ} \mathrm{C} 20 \mathrm{~s}, 56^{\circ} \mathrm{C} 20 \mathrm{~s}$, and $72^{\circ} \mathrm{C} 20 \mathrm{~s}$ and final extension at $94^{\circ} \mathrm{C} 20 \mathrm{~s}, 56^{\circ} \mathrm{C} 20 \mathrm{~s}$, and $72^{\circ} \mathrm{C} 6 \mathrm{~min}$. The final products were visualized by EtBr staining. The PCR fragments from $\mathrm{Nrf2}^{+/+}$ and $\mathrm{Nrf} 2^{-/-}$alleles were 262 and 214 bps, respectively (fig. 1).

\section{Ambient Particle Collection and Endotoxin Detection}

The ambient UFP $(<0.18 \mu \mathrm{m})$ for nasal allergic sensitization were collected in downtown Los Angeles using the Versatile Aerosol Concentration Enrichment System as previously described [19]. Analyses of the chemical composition, intrinsic oxidant potential and endotoxin levels of these particles have been described previously [19].

\section{Intranasal Allergic Sensitization and Particle Exposure}

Endotoxin-free OVA was prepared using the commercial endotoxin removal columns. Intranasal allergic sensitization was achieved following the protocol that we previously described [19]. Briefly, on day 1 and under anesthesia by ketamine $(50 \mathrm{mg} / \mathrm{kg}) /$ xylazine $(10 \mathrm{mg} / \mathrm{kg})$ the mice in the OVA/UFP exposure group received intranasal instillation of $0.5 \mu \mathrm{g}$ of UFP alone in a total volume of $50 \mu \mathrm{l}$. Mice in the OVA only and control groups received the same volume of saline. On days 2, 4, 7 and 9, mice in the OVA/ UFP group received intranasal instillation of the particles as described above and $10 \mu \mathrm{g}$ of OVA, while those in the OVA-only and control groups received $10 \mu \mathrm{g}$ of OVA and $50 \mu \mathrm{l}$ of saline, respectively. After 2 weeks all animals received a 30-min challenge of aerosolized OVA $(1 \% \mathrm{w} / \mathrm{v})$ for 2 consecutive days following which animals were sacrificed $48 \mathrm{~h}$ later [19]. Each group included at least 6 animals.

\section{Preparation and Stimulation}

DC were prepared from bone marrow-derived hematopoietic cells of $\mathrm{Nrf}_{2}^{+/+}$and $\mathrm{Nrf}^{-/-}$mice using the protocol previously described by Williams et al. [16]. Briefly, bone marrow precursor cells were collected from the femora and tibiae of female mice and cultured in RPMI 1640 medium containing 20 mM HEPES buffer, $2 \mathrm{mM} \mathrm{L}$-glutamine, $2.5 \mu \mathrm{g} / \mathrm{ml}$ penicillin/streptomycin/amphotericin B and $10 \%(\mathrm{v} / \mathrm{v}) \mathrm{FBS}$ at a cellular density of $5 \times 10^{5}$ cells per well

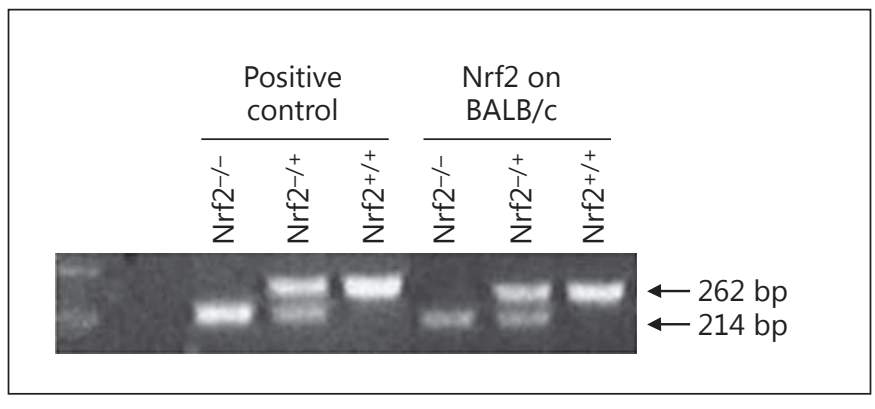

Fig. 1. Nrf2 genotyping by PCR. Deletion of Nrf2 in BALB/c mice was confirmed by genotyping. PCR fragments of 262 and 214 bp represent $\mathrm{Nrf}^{+/+}$and $\mathrm{Nrf}_{2}{ }^{-/}$alleles, respectively.

in 6-well tissue culture plates in a total volume of $4 \mathrm{ml}$. Cultures were pulsed on day 0 and every $48 \mathrm{~h}$ with a combination of GMCSF (25 ng/ml) and IL-4 (10 ng/ml) [16]. On day 8 immature DC were harvested, washed, and plated at a density of $1.5 \times 10^{6}$ cells/ $\mathrm{ml}$ in 12-well plates for stimulation. Due to the low UFP concentration $(10 \mu \mathrm{g} / \mathrm{ml}$ in distilled water), cell culture medium for UFP stimulation was prepared by using UFP suspension to reconstitute commercially available RPMI 1640 medium powder following the manufacture's instruction. This culture medium was further supplemented with $10 \%$ FBS, $1 \%$ penicillin/streptomycin/ amphotericin B and $5 \mathrm{ng} / \mathrm{ml} \mathrm{GM-CSF}$. The control medium was prepared with distilled water in place of UFP suspension.

\section{Allergic Sensitization by Intratracheal Adoptive Transfer of $D C$}

DC from $\mathrm{Nrf}_{2}^{+/+}$and $\mathrm{Nrf}^{-/-}$mice were stimulated with endotoxin-free OVA $(50 \mu \mathrm{g} / \mathrm{ml})$ with or without UFP $(10 \mu \mathrm{g} / \mathrm{ml})$ for $16 \mathrm{~h}$. Cells in the control group received equal volume of vehicle alone. Loosely attached cells were collected and thoroughly washed 3 times with cold PBS to remove OVA and UFP before being resuspended in cold PBS at $2 \times 10^{7}$ cells $/ \mathrm{ml}$. After recipient BALB/c mice were anesthetized by a combination of ketamine $(100 \mathrm{mg} /$ $\mathrm{kg}) / x y$ lazine $(10 \mathrm{mg} / \mathrm{kg})$, adoptive transfer was achieved by intratracheal instillation of $10^{6} \mathrm{Nrf} 2^{+/+}$or $\mathrm{Nrf2}^{-/-} \mathrm{DC}$ in a total volume of $50 \mu \mathrm{l}$. Fourteen days after the adoptive transfer, the mice were challenged with OVA aerosol ( $1 \% \mathrm{w} / \mathrm{v}, 30 \mathrm{~min})$ for 3 consecutive days and necropsy was conducted $48 \mathrm{~h}$ after the final OVA inhalation. Each group included at least 6 mice.

\section{Animal Necropsy, Sample Collection and Analysis}

Mice were sacrificed by intraperitoneal injection of pentobarbital. Blood and bronchoalveolar lavage (BAL) collection and differential BAL cell counts were performed as previously described [19]. The right lung was collected and stored in liquid nitrogen for future analyses. The left lung was expanded with $10 \%$ buffered formalin before being harvested for histological analyses. Plasma OVA-specific IgG1 (OVA-IgG1) and IgE (OVA-IgE) were measured by ELISA [19]. The levels of IL-13 in the BAL fluid were determined using commercially available ELISA kit according to the manufacturer's instructions. 

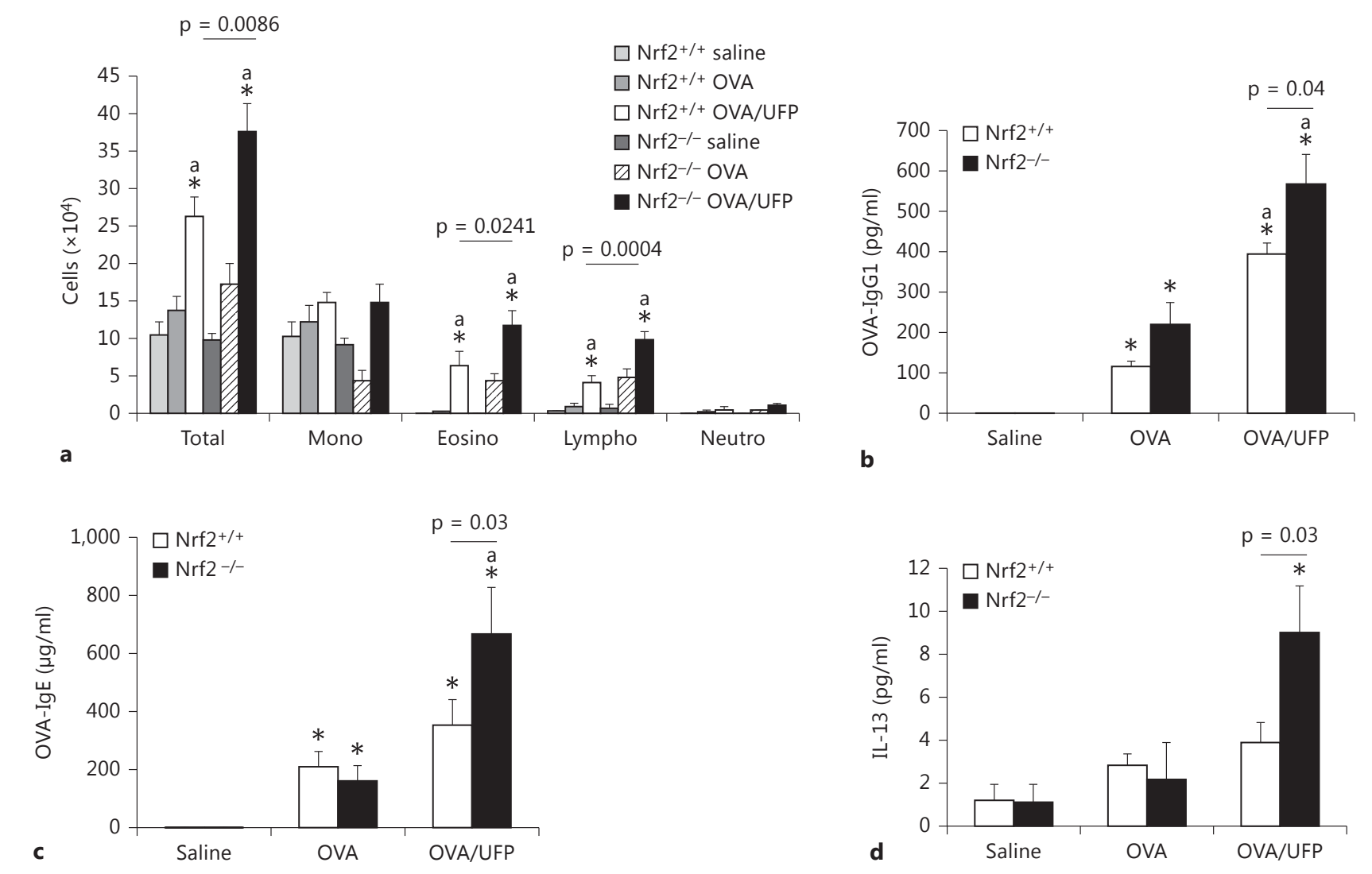

Fig. 2. The adjuvant effect of intranasally administered ambient UFP on OVA sensitization was further enhanced in $\mathrm{Nrf} 2^{-/-}$mice. a Increased BAL cell count including total cell number, eosinophils and lymphocytes; ${ }^{*} \mathrm{p}<0.02$ compared with respective saline control, ${ }^{a} \mathrm{p}<0.05$ compared with respective OVA. b Elevated OVA-IgG1 level in the blood; ${ }^{*} \mathrm{p}<0.01$ compared with respective

\section{Lung Histology}

Histopathological analysis of the lung was performed as previously described [20]. Formalin-inflated lung tissue was embedded in paraffin, sectioned and stained by $\mathrm{HE}$, also as previously described [20].

\section{Analysis of DC Response to UFP Stimulation}

DC response to stimulation with UFP was assessed by analyzing cell surface expression of functional molecules (MHC class II, CD80, CD86 and CD40) and cytokine production (IL-12p70, IL-6 and IL-1 $\beta$ ). The expression of cell surface-associated functional molecules was determined by flow cytometry using FITC-labeled fluorescent anti-MHC class II, anti-CD80, anti-CD86 and antiCD40 antibodies combined with PE-labeled anti-CD11c antibody as previously described [21]. Cytokine analysis was performed using commercial ELISA kits. saline control, ${ }^{\mathrm{a}} \mathrm{p}<0.01$ compared with respective OVA. c Increased OVA-IgE production; ${ }^{*} \mathrm{p}<0.01$ compared with respective saline control, a $\mathrm{p}<0.05$ compared with respective OVA. d Increased IL-13 level in the BAL fluid; ${ }^{*} \mathrm{p}<0.05$ compared with all other groups.

\section{Statistical Analysis}

Data are expressed as mean \pm SE. Differences among groups were evaluated by analysis of variance and the Student $t$ test was used to distinguish between pairs of groups. We considered an alpha value of $\mathrm{p}<0.05$ as statistically significant [19].

\section{Results}

\section{The Adjuvant Effect of Ambient UFP Was}

Significantly Enhanced in Nrf2 $2^{-/-}$Mice

Since it has been established that Nrf2 plays a key role in protecting against the proinflammatory effect of PM in the respiratory system, we first sought to investigate the role of Nrf2 in mediating the adjuvant effect of ambient UFP on allergic sensitization to OVA. 
$\mathrm{Nrf}^{+/+}$and $\mathrm{Nrf}^{-/-}$mice on BALB/c background were intranasally sensitized by endotoxin-free OVA $(10 \mu \mathrm{g})$ with or without ambient UFP $(0.5 \mu \mathrm{g})$ and challenged by OVA aerosol (1\%) as described in Materials and Methods. BAL analysis showed that while OVA/UFP effectively induced an increase in the eosinophil count of both $\mathrm{Nrf} 2^{+/+}$and $\mathrm{Nrf2}^{-/-}$mice compared to the animals sensitized by saline or OVA alone, the number of eosinophils from $\mathrm{Nrf}^{-/-}$animals was significantly greater than that from $\mathrm{Nrf}^{+/+}$mice in the same treatment group (fig. 2a). Analysis of OVA-specific antibodies in the plasma showed that sensitization by OVA alone increased OVA-IgG1 production in $\mathrm{Nrf2}^{+/+}$as well as $\mathrm{Nrf2} 2^{-/-}$mice. While UFP further increased OVA-IgG1 production in both types of animals, the level of this antibody was significantly higher in $\mathrm{Nrf}^{-/-}$mice compared to their $\mathrm{Nrf}^{+/+}$counterpart (fig. 2b). OVA sensitization also elevated the levels of OVA-IgE in both $\mathrm{Nrf}_{2}^{+/+}$and $\mathrm{Nrf}^{-/-}$mice compared with respective saline controls. However, the enhancing effect of UFP on OVA-IgE production was only observed in $\mathrm{Nrf}^{-/-}$animals (fig. 2c). Moreover, UFP-induced allergic airway inflammation was also accompanied by increased IL-13 secretion in the BAL fluid from $\mathrm{Nrf}^{-/-}$ mice sensitized by OVA/UFP, a signature Th2 cytokine response for allergic inflammation (fig. 2d). Histological analysis showed that only $\mathrm{Nrf}^{-/-}$mice exposed to OVA/ UFP developed a prominent inflammation in the small airways of the lung (fig. 3). These results show that although UFP exerted an enhancing effect on OVA-induced allergic sensitization in both $\mathrm{Nrf}^{+/+}$and $\mathrm{Nrf2}^{-/-}$ mice as evidenced by increased BAL eosinophil and lymphocyte counts and plasma OVA-IgG1 level, the significantly higher levels of these endpoints in $\mathrm{Nrf} 2^{-/}$ mice ( $\mathrm{p}=0.02$ for eosinophils; $\mathrm{p}=0.0004$ for lymphocytes; $\mathrm{p}=0.04$ for OVA-IgG1) compared with $\mathrm{Nrf}^{+/+}$ animals suggest that lack of Nrf2 may further augment this effect. Moreover, that UFP increased OVA-IgE and IL-13 production only in Nrf2 ${ }^{-/-}$mice provides clear evidence to support this suggestion.

Exposure of DC to UFP Led to a Cytokine Milieu That May Polarize the Immune Response

To determine the role of Nrf2 in mediating the response of DC to ambient UFP, we investigated the direct impact of these particles on DC from $\mathrm{Nrf}_{2}^{+/+}$and $\mathrm{Nrf}^{-/-}$ mice. DC were stimulated with UFP $(10 \mu \mathrm{g} / \mathrm{ml})$ for $16 \mathrm{~h}$ before being analyzed for the expression of MHC class II as a surrogate maturation marker and costimulatory molecules (CD80, CD86 and CD40). Flow cytometric analysis revealed that UFP increased the expression of MHCII
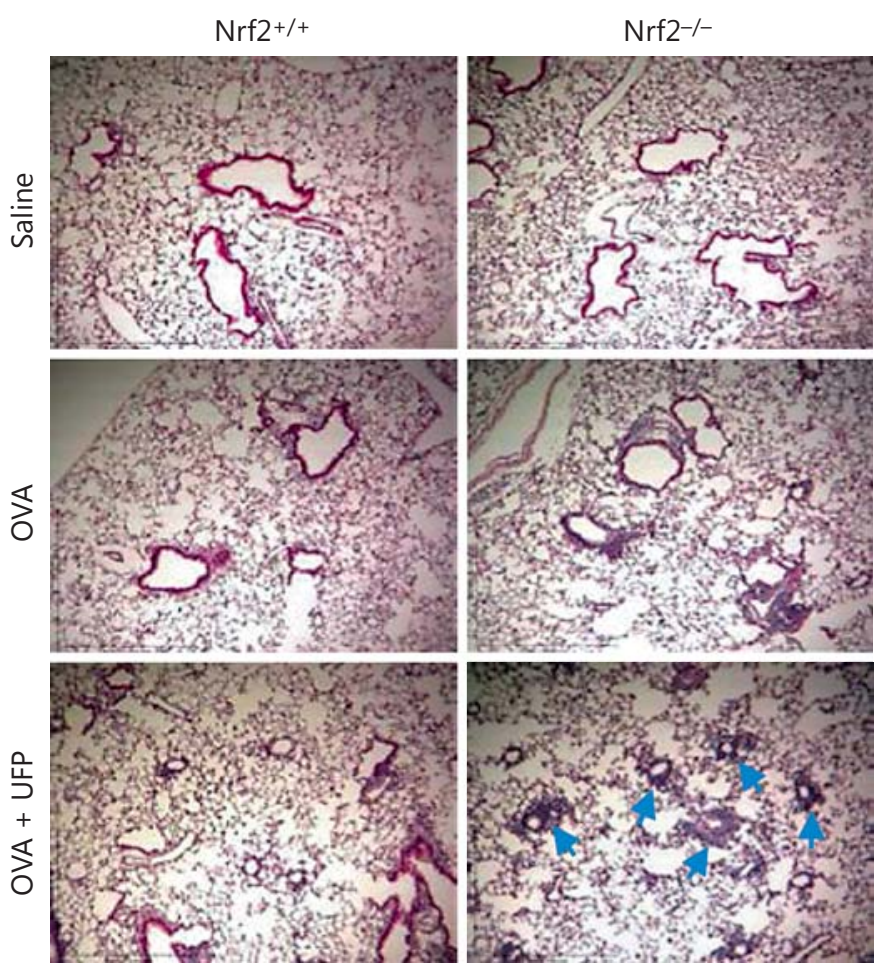

Fig. 3. Histological analysis showing that $\mathrm{Nrf} 2^{-/-}$mice developed a more severe inflammation in the lung when exposed to UFP during OVA sensitization and smaller airways were more involved (indicated by arrows).

on the surface of CD11c+ DC from both $\mathrm{Nrf}^{+/+}$and $\mathrm{Nrf2}^{-/-}$animals, indicating that these particles were capable of inducing DC maturation (fig. 4a). Direct exposure to UFP also led to increased expression of costimulatory molecules CD80 (fig. 4b) and CD86 (fig. 4c) on $\mathrm{Nrf}_{2}^{+/+}$and $\mathrm{Nrf2}^{-/-}$DC surface, whereas it had no effect on the expression of CD40 (data not shown). The extent of UFP-induced surface marker expression was similar between $\mathrm{Nrf}^{+/+}$and $\mathrm{Nrf}^{-/-}$DC.

Studies have found that DC exposed to PM could produce a cytokine environment that favors Th2 immunity [17]. Because we did not observe any difference in the UFP effect on surface molecule expression between $\mathrm{Nrf}_{2}^{+/+}$and $\mathrm{Nrf}^{-/-}$DC, we asked whether UFP could induce different cytokine profiles. To answer this question, the media from the same cell cultures were analyzed for the levels of three cytokines (IL-12p70, IL-6 and IL-1 $\beta$ ) that were relevant to allergic airway inflammation.

Our results showed that under resting condition there was a significant difference in the level of IL-12p70 between $\mathrm{Nrf2}^{+/+}$and $\mathrm{Nrf}_{2}{ }^{-/}$DC. While untreated $\mathrm{Nrf2}^{+/+}$ 


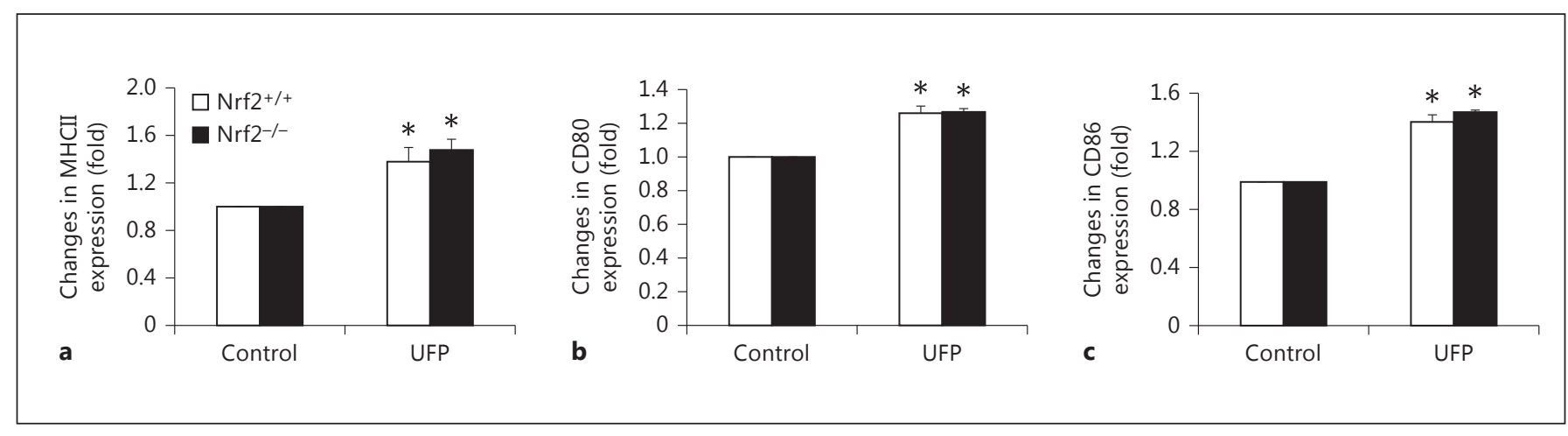

Fig. 4. UFP increased functional molecule expression on $\mathrm{Nrf2} 2^{+/+}$and $\mathrm{Nrf} 2^{-/-}$DC surface in a similar fashion. Surface molecule expression was analyzed by flow cytometry as described in Materials and Methods. a MHC II. b CD80. c CD86. * $\mathrm{p}<0.01$ compared with respective control.

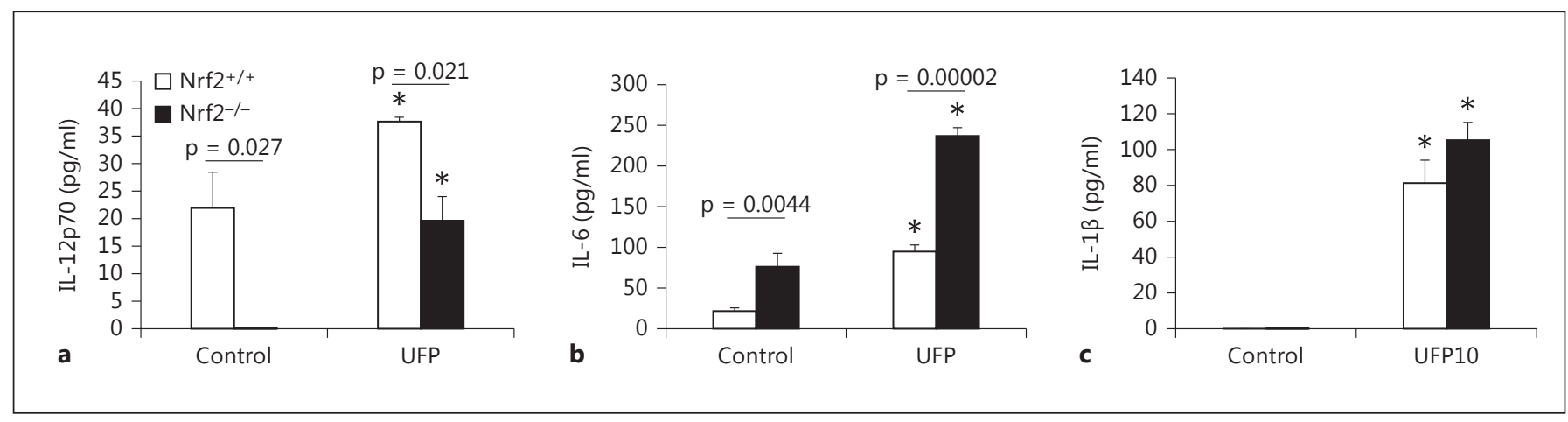

Fig. 5. The effect of UFP on cytokine production by $\mathrm{Nrf}_{2}^{+/+}$and $\mathrm{Nrf}^{-/-}$DC after 16-hour exposure. Cytokine levels in the DC culture media were measured by ELISA as described in Materials and Methods. a IL-12p70; ${ }^{*} \mathrm{p}<0.05$ compared with respective control. b IL-6; * $\mathrm{p}<0.01$ compared with respective control. c IL-1 $\beta$; $^{*} \mathrm{p}<$ 0.001 compared with respective control.

DC maintain a basal level of IL-12p70, $\mathrm{Nrf2}^{-/-}$DC failed to produce this cytokine (fig. 5a). Although UFP stimulation significantly increased IL- 12 p70 production by $\mathrm{Nrf2}^{-/-}$DC, the level of this cytokine remained significantly lower as compared to their $\mathrm{Nrf2}^{+/+}$counterparts (fig. 5a). In contrast, unstimulated $\mathrm{Nrf2}^{-/-}$DC produced greater levels of IL-6 than Nrf2 ${ }^{+/+}$DC (fig. 5b). Even though UFP stimulation increased IL- 6 production by both $\mathrm{Nrf}^{+/+}$and $\mathrm{Nrf}^{-/-}$DC, the level of IL-6 in Nrf2 $2^{-/-}$ culture medium was still significantly higher than that from $\mathrm{Nrf}^{+/+}$DC (fig. 5b). UFP also induced IL-1 $\beta$ production by DC, but there was no significant difference between $\mathrm{Nrf}_{2}^{+/+}$and $\mathrm{Nrf}^{-/-} \mathrm{DC}$ (fig. 5c). Taken together, these data suggest that while UFP had similar stimulatory effect on the cell surface expression of functional molecules by both $\mathrm{Nrf}_{2}^{+/+}$and $\mathrm{Nrf2} 2^{-/-}$DC, Nrf2 deficiency alone was capable of creating a unique cytokine environment that could promote an altered $\mathrm{T}$ cell response.

\section{Absence of Nrf2 in DC Enhanced the Adjuvant Effect of UFP on Allergic Sensitization}

DC play a central role in regulating the immune response. It has been demonstrated that allergic sensitization by intratracheal adoptive transfer of OVA-pulsed DC into recipient mice could lead to allergic inflammation in the lung after secondary OVA challenge [22]. To determine whether different responses of $\mathrm{Nrf2}^{+/+}$and $\mathrm{Nrf2}^{-/-}$ DC to UFP could be reflected as different adjuvant activity in vivo, we sensitized wild-type $\mathrm{BALB} / \mathrm{c}$ recipient mice with $\mathrm{Nrf}_{2}^{+/+}$and $\mathrm{Nrf2}^{-/-} \mathrm{DC}$ that had been first stimulated or primed by saline, OVA $(50 \mu \mathrm{g} / \mathrm{ml})$ or OVA $(50 \mu \mathrm{g} / \mathrm{ml}) /$ UFP $(10 \mu \mathrm{g} / \mathrm{ml})$. Upon secondary OVA challenge, animals 


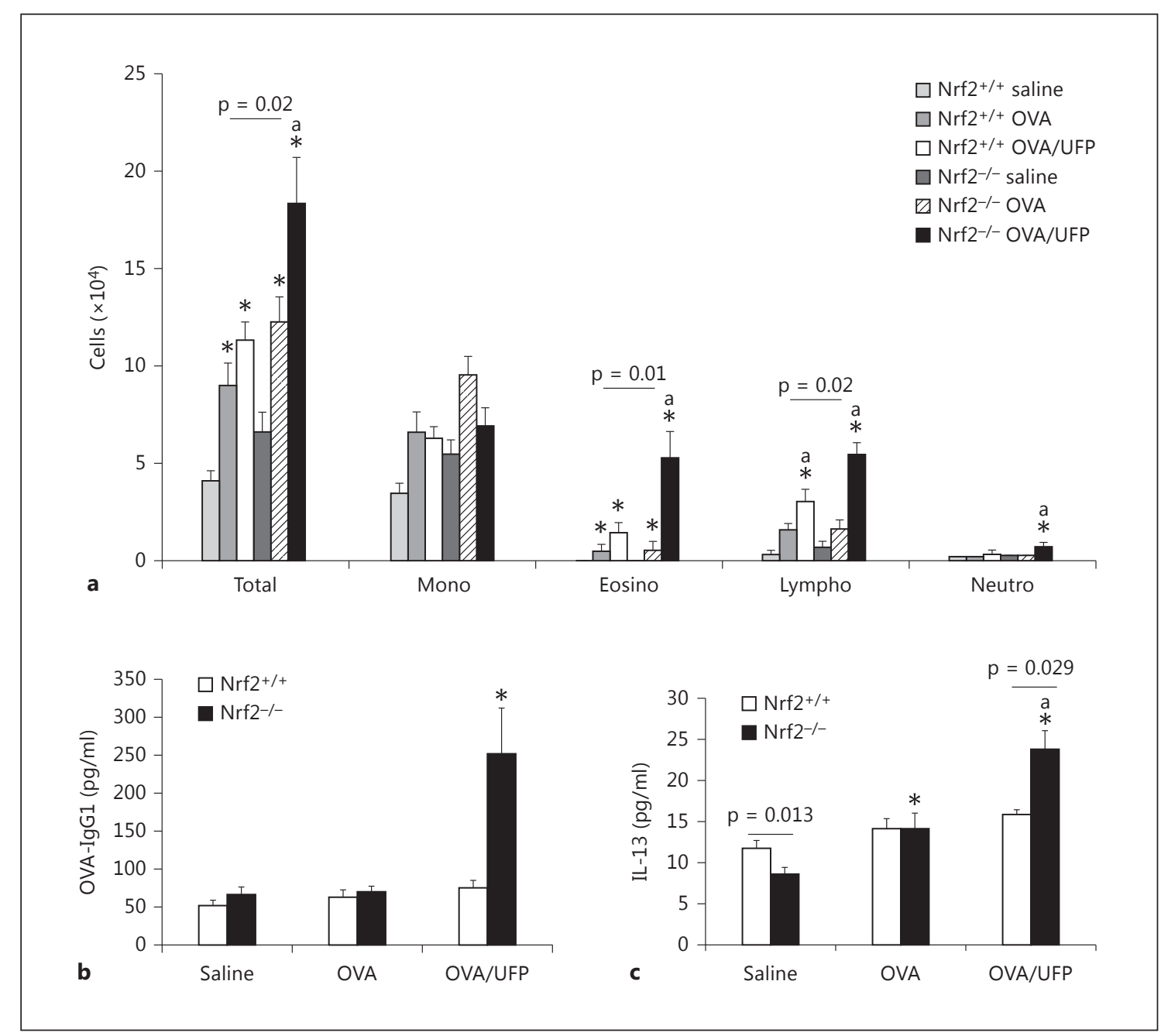

Fig. 6. Recipient mice developed a stronger allergic airway inflammation when sensitized by OVA/UFP-exposed $\mathrm{Nrf}^{-/-}$DC via adoptive transfer. Adoptive transfer and secondary OVA aerosol challenge were performed as described in Materials and Methods. a BAL cellular differentiation showing that sensitization by OVA/ UFP-treated $\mathrm{Nrf}^{-/-}$DC led to eosinophilic inflammation in the lungs; ${ }^{*} \mathrm{p}<0.01$ compared with respective saline control, ${ }^{\mathrm{a}} \mathrm{p}<0.01$ compared with respective OVA group. b Increased OVA-IgG1 in the blood of recipient mice sensitized by OVA/UFP-exposed DC; $* \mathrm{p}<0.02$ compared with all other groups. c Elevated IL-13 level in the BAL fluid; ${ }^{*} \mathrm{p}<0.01$ compared with respective saline control, ${ }^{\mathrm{a}} \mathrm{p}<0.05$ compared with respective OVA group. sensitized by OVA/UFP-primed Nrf2 ${ }^{-/-}$DC developed a more significant allergic inflammatory response in their lungs as compared to those DC in all other treatment groups. Analysis of infiltrating BAL cell differentiation showed that sensitization by OVA- or OVA/UFP-treated $\mathrm{Nrf2}^{+/+}$DC increased the numbers of eosinophils in the lungs of recipient mice compared to the animals sensitized by control DC, but we found no statistically significant difference between these two groups (fig. 6a).

By contrast, while sensitization by OVA- and OVA/ UFP-treated $\mathrm{Nrf2}^{-/-}$DC also increased BAL eosinophil infiltration in recipient mice, OVA/UFP-treated $\mathrm{Nrf}^{-/-}$ DC caused a further increase in BAL eosinophil count (fig. 6a). Adoptive transfer of OVA/UFP-treated Nrf2 $2^{+/+}$ and $\mathrm{Nrf}^{-/-}$DC also increased BAL lymphocyte count compared with respective control and OVA-treated groups; this effect was markedly significant in mice that had received Nrf2 ${ }^{-/-}$DC (fig. 6a). Consistent with the differential BAL cellular data, OVA-IgG1 level in the blood was also significantly higher in mice sensitized by OVA/ UFP-exposed $\mathrm{Nrf2}^{-/-}$DC, whereas it remained unchanged in animals sensitized by $\mathrm{Nrf}^{+/+} \mathrm{DC}$ (fig. 6b). We 
also measured OVA-IgE in the blood, but this was undetectable by our ELISA. While sensitization by $\mathrm{Nrf2}^{+/+} \mathrm{DC}$ exposed to either OVA alone or OVA/UFP had little impact on IL-13 production in the lungs of recipient animals, OVA-treated $\mathrm{Nrf2}^{-/-}$DC significantly increased IL13 production in the lung, which was further increased in the presence of UFP (fig. 6c). Histological analysis of the lung showed that animals sensitized by OVA/UFP-treated $\mathrm{Nrf}^{-/-}$DC developed a significant lung inflammation (fig. 7f), whereas mice that received $\mathrm{Nrf}^{+/+} \mathrm{DC}$ in the same treatment group only had a minimal inflammatory response (fig. 7c). Moreover, animals sensitized by OVA/ UFP-exposed $\mathrm{Nrf2}^{-/-} \mathrm{DC}$ also had multiple inflammatory foci along the conducting airways (fig. $7 \mathrm{~g}$ ).

Taken together, these data showed that allergic sensitization by adoptive transfer of OVA/UFP-treated DC could yield an in vivo outcome similar to that in the intranasal sensitization model, including increased eosinophil count and IL-13 level in the BAL, and significantly enhanced OVA-IgG1 production. The only difference was that while direct intranasal exposure to OVA/UFP led to an OVA-IgE response in $\mathrm{Nrf}^{-/-}$mice, we were unable to detect this antibody in the recipient animals sensitized by adoptive transfer (see Discussion). Nonetheless, the results from our adoptive transfer study provided evidence that lack of functional Nrf2 in DC could markedly strengthen the adjuvant effect of ambient UFP on allergic sensitization.

\section{Discussion}

In the present work, we began with assessing the role of Nrf2 in mediating the adjuvant activity of ambient UFP on OVA sensitization using the intranasal sensitization mouse model previously reported by us [19]. We found that the adjuvant effect of UFP was significantly enhanced in $\mathrm{Nrf2}^{-/-}$mice compared with $\mathrm{Nrf2}{ }^{+/+}$animals. To determine whether UFP exert their adjuvant effect by interfering with DC function, we then analyzed the impact of these particles on DC functional molecule expression and cytokine production. Our results showed that while UFP induced similar increases in the expression of MHC class II, CD80 and CD86 on both Nrf2 ${ }^{+/+}$and Nrf2 $2^{-/-}$DC surface, it had different effects on DC cytokine production. $\mathrm{Nrf2}^{-/-}$DC consistently had lower levels of IL-12p70 and produced more IL- 6 than Nrf2 ${ }^{+/+}$DC. Finally, to confirm that these unique features in $\mathrm{Nrf}^{-/-}$DC were responsible, at least in part, for the enhanced adjuvant activity that was observed in $\mathrm{Nrf}^{-/-}$animals from our intranasal sensitiza- tion study, we performed a proof-of-principle study using intratracheal adoptive transfer of $\mathrm{Nrf}_{2}^{+/+}$or $\mathrm{Nrf} 2^{-/-}$ DC that had been treated with saline, OVA or OVA/UFP for allergic sensitization. Our data demonstrated that upon secondary OVA aerosol challenge recipient animals sensitized by OVA/UFP-treated Nrf2 ${ }^{-/-}$DC developed a significantly stronger allergic inflammation in the lung, confirming that Nrf2 deficiency in DC could indeed enhance the adjuvant effect of UFP on allergic sensitization.

Previously published studies have shown that Nrf2 plays an important role in protecting against lung inflammation including that associated with exposure to environmental agents [9-12, 16, 23-25]. Detailed characterization of ambient UFP used for this study showed that these particles contained a high PAH content and had a strong oxidant potential to induce cellular oxidative stress as well as enhance OVA sensitization in vivo [19]. In the present study, we obtained similar results by showing that UFP acted as an adjuvant for OVA sensitization in $\mathrm{Nrf2}^{+/+}$ mice.

One of the most important findings here was the clear demonstration that the adjuvant effect of these prooxidative UFP was further enhanced in $\mathrm{Nrf2}^{-/-}$mice (fig. 2, 3). While other studies have reported that repeated exposure to low-dose DEP over an 8-week period enhanced OVAinduced allergic airway inflammation in $\mathrm{Nrf}^{-/-}$mice, the significant feature of this study is that we were able to demonstrate the importance of Nrf2 in mediating the specific effect of ambient UFP on the primary immune response $[10,11]$. Many enzymes regulated by Nrf2 are involved in maintaining cellular redox homeostasis and detoxifying xenobiotics including the organic compounds associated with ambient UFP $[2,4,6]$. We provide evidence linking the adjuvant effect of ambient UFP to the PAH content of those particles [19]. Thus, the more severe allergic inflammation that we observed in the lungs of $\mathrm{Nrf}_{2}{ }^{-/}$mice was likely the result of a failure to metabolize UFP-associated organic xenobiotics (e.g. PAH and quinones) and restore cellular redox balance. Nrf2 regulates a large number of antioxidant and phase II enzymes including glutathione S-transferase and NADPH quinone-oxidoreductase-1 [5]. Genetic polymorphisms in such enzymes have been linked to increased susceptibility to air pollution-induced asthma exacerbation [26-29]. In this regard, our findings enhance our understanding of gene-environment interactions that determine an individual's susceptibility to the adverse respiratory effects of airborne PM such as ambient UFP.

The much higher level of IL-13 in the BAL fluid from $\mathrm{Nrf2}^{-/-}$mice that were intranasally sensitized by OVA/ 

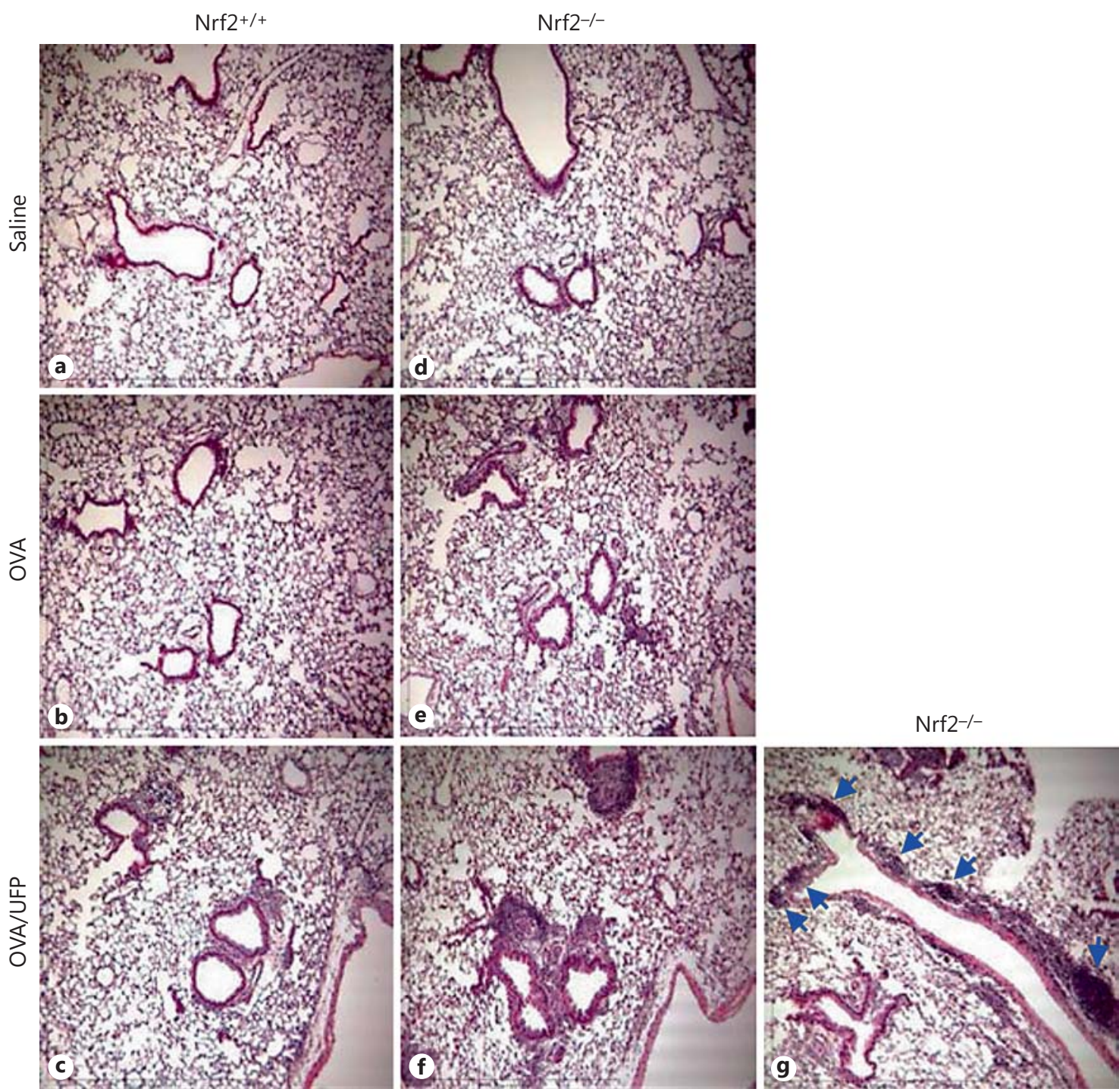

Fig. 7. a-g Lung histology showing a severe inflammation in the mice sensitized by OVA/UFP-pulsed Nrf2 ${ }^{-/-}$ DC. Note the multiple inflammatory foci along the conducting airway $(\mathbf{g})$.

UFP indicated that Nrf2 deficiency favored a Th2 immune response (fig. 2d). Since DC are important in orchestrating $\mathrm{T}$ cell differentiation we sought to determine whether the enhanced adjuvant effect of UFP in $\mathrm{Nrf}_{2}^{-/-}$ mice was due to the lack of this transcription factor in DC. Using UFP to stimulate DC in vitro, we showed that UFPinduced increases in the expression of MHCII, CD80 and CD86 were similar between $\mathrm{Nrf}^{+/+}$and $\mathrm{Nrf}^{-/-} \mathrm{DC}$. This suggested that upregulation of DC functional molecules might not be the determining factor for the stronger adjuvant activity of UFP in Nrf2 ${ }^{-/-}$mice (fig. $4 \mathrm{a}-\mathrm{c}$ ). Several published studies have demonstrated that PM such as
DEP and PM2.5 could promote DC to create a Th2 cytokine environment and this effect was stronger in the absence of Nrf2 $[16,17]$. To determine whether the lack of Nrf2 in DC could lead to a different cytokine profile that might explain the stronger adjuvant effect of UFP on $\mathrm{Nrf} 2^{-/-}$mice, we measured the levels of three DC cytokines (IL-12p70, IL- 6 and IL-1 $\beta$ ) in the same culture media (fig. $5 a-c)$. The absence of IL-12p70 and higher level of IL- 6 produced by $\mathrm{Nrf2}^{-/-}$DC under resting conditions suggested that Nrf2 deficiency alone might polarize DC response, that is to say behaving like a pro-Th2 DC. This possibility was further supported by the fact that although 
UFP increased the production of IL-12p70 and IL- 6 by DC, the same trend was still observed in $\mathrm{Nrf}^{-/-}$DC. IL12 directs Th1 differentiation by activating transcription factor STAT4 [18, 30]. Absence of IL-12 signaling could favor Th2 differentiation, which is one of the major immunological bases of allergic airway inflammation [30]. In contrast, it has been demonstrated that adoptive transfer of DC from mycobacteria-infected mice could successfully block ragweed-induced eosinophilia and mucus production through a mechanism involving IL-12 [11, 31].

Our data were in keeping with a number of studies which have shown that PM (e.g. PM2.5 and DEP) could have a pro-Th2 effect on DC by either promoting Th2 cytokine production or suppressing IL-12, and this effect is further strengthened when Nrf2 was deleted [13, 16, 32, 33]. IL-6 is another cytokine that is linked to allergic asthma in mice and was found in this study to be produced at different levels by $\mathrm{Nrf2}^{+/+}$and $\mathrm{Nrf2} 2^{-/-}$DC (fig. 5b) [34]. It has been suggested that IL- 6 induces the expansion of Th2 effector cells through itsinteraction with CD4+CD25+ regulatory T cells (T-reg) [31, 32]. T-reg reduces the production of DC-derived proinflammatory cytokines including IL- 6 and IL- $1 \beta$ rendering these cells ineffective in stimulating T cell proliferation [35]. IL- 6 released by DC has been found to be capable of inhibiting T-reg-mediated peripheral tolerance $[34,35]$. In addition, IL- 6 from antigen-presenting cells (APC) could also induce the initial production of IL-4 in naïve T cells [36]. Thus, the constitutively high level of IL- 6 combined with a low level of IL-12 in Nrf2 $2^{-/}$DC may 'prime' these APC towards a polarized immune response, which was further strengthened in the presence of UFP. Finally, our cytokine analysis also demonstrated that UFP-induced IL- $1 \beta$ release from $\mathrm{Nrf2}^{+/+}$and $\mathrm{Nrf2}^{-/-}$DC with similar potency (fig. 5c). IL-1 $\beta$ plays an important role in airway inflammation [37]. Release of IL- $1 \beta$ from DC involves NALP3 inflammasome/Caspase-1 pathway after these cells sense 'danger signals', including particulate adjuvant and reactive oxygen species [38]. However, other studies also suggest the presence of a NALP3/Caspase-1-independent mechanism for IL- $1 \beta$ production such as in the case of DEP-induced lung inflammation [39]. Whether UFP-induced IL- $1 \beta$ release from DC observed in this study was mediated through NALP3 inflammasome activation is unclear at this time, but our results suggested that Nrf2 might not be essential for this effect of UFP.

To test the hypothesis that the adjuvant effect of ambient UFP on allergic sensitization took place at DC level and the lack of Nrf2 in DC could further strengthen this effect, we performed adoptive transfer studies to sensitize wild-type BALB/c mice with $\mathrm{Nrf2} 2^{+/+}$or $\mathrm{Nrf2}^{-/-}$DC that had been stimulated with saline, OVA or OVA/UFP (fig. 6a-c, 7). We found that upon secondary OVA challenge, mice sensitized by OVA/UFP-stimulated $\mathrm{Nrf}^{-/-}$ DC developed a much stronger allergic inflammation as measured by increased BAL eosinophil count and plasma OVA-IgG1 level compared with animals in all other groups. This was accompanied by increased IL-13 production in the lung. Although OVA-IgE was not detectable by our ELISA, the significant increase of OVA-IgG1 in animals sensitized by OVA/UFP-treated $\mathrm{Nrf}^{-/-}$DC was consistent with the allergic inflammation in their lungs. It is highly likely that this enhanced allergic airway inflammation in recipient mice sensitized by OVA/UFPtreated $\mathrm{Nrf}^{-/-} \mathrm{DC}$ was the result of a unique Th2-favoring cytokine milieu (low level of IL-12p70 and high level of IL-6) created by the absence of Nrf2 in DC. The absence of OVA-IgE response may be explained by the possibility that while sensitization by adoptive transfer of OVA/UFP-stimulated DC was able to elicit eosinophilic inflammation and increase the production of IL-13 and OVA-IgG1, a single adoptive transfer of DC for allergic sensitization was not potent enough to induce an IgE response.

In both our intranasal sensitization and adoptive transfer studies the increase of IL-13 is consistently associated with the absence of Nrf2 and exposure to OVA/ UFP (fig. 2d, 6c). While it has been established that murine IL- 13 is produced by Th 2 cells during allergic inflammation, nuocytes, a recently recognized innate immune cell population, have been found to play an important role in Th2 immunity [40]. Nuocytes produce IL-5 and IL-13 in response to IL-25 and IL-33 released by airway epithelial cells and contribute to eosinophilia and goblet cell metaplasia during allergic airway inflammation [40]. Currently there is little information about the role of $\mathrm{Nrf} 2$ in regulating nuocyte function. In our case we do not exclude the possibility that $\mathrm{Nrf2}^{-/-}$nuocytes may act synergistically (i.e. through their IL-13 production) with $\mathrm{Nrf2}^{-/-}$DC to further strengthen the adjuvant effect of UFP on OVA sensitization.

Numerous studies have explored the role of Nrf2 in protecting against allergic airway inflammation associated with DEP or PM2.5 exposure, yet there remains a paucity of experimental evidence of the role played by ambient UFP. To our knowledge, this is the first study to provide direct evidence, through the use of $\mathrm{Nrf}^{-/-} \mathrm{DC}$ and adoptive transfer approaches, to show that the adjuvant effect of ambient UFP on allergic sensitization starts 
at the level of DC and that Nrf2 in these cells is an important factor in determining the severity of allergic lung inflammation associated with UFP exposure in our model.

In summary, our study demonstrates the importance of Nrf2 in mediating the adjuvant effect of ambient UFP on allergic sensitization. We have shown that $\mathrm{Nrf}^{-/-}$enabled DC to promote a cytokine milieu that favored Th2 polarization - even under resting conditions. This suggests that even without any exogenous stimuli, the mere absence of Nrf2 in DC may interfere with the normal physiological functions of innate immunity. While our findings assist our understanding of how Nrf2 protects against the enhancing effect of ambient UFP on allergic airway inflammation, there are many unanswered questions. One important question is how Nrf2 regulates the interaction between innate and adaptive immunity. More detailed studies should assist our ability to develop more effective preventive and treatment strategies for the clinical management of air pollution-associated allergic disease such as asthma and allergic rhinitis.

\section{Acknowledgments}

This study was supported by NIH Grants U19 AI-070453, US EPA STAR Award RD-83241301, EPA grant EPA-G2006-STARQ1, Southern California Environmental Health Sciences Center Pilot Project Grant H44764 and funding from the AQMD Board of Southern California Particle Center.

\section{Disclosure Statement}

The research described in this article has been reviewed by the National Health and Environmental Effects Research Laboratory, US Environmental Protection Agency, and approved for publication. Approval does not signify that the contents necessarily reflect the views and policies of the agency, nor does the mention of trade names or commercial products constitute endorsement or recommendation for use. Additionally, the views and opinions of authors expressed herein do not necessarily state or reflect those of the United States Government or of The US Environmental Protection Agency.

\section{References}

1 Di Giampaolo L, Quecchia C, Schiavone C, Cavallucci E, Renzetti A, Braga M, Di Gioacchino M: Environmental pollution and asthma. Int J Immunopathol Pharmacol 2011;24: 31S-38S.

-2 Li N, Xia T, Nel AE: The role of oxidative stress in ambient particulate matter-induced lung diseases and its implications in the toxicity of engineered nanoparticles. Free Radic Biol Med 2008;44:1689-1699.

3 Riedl MA, Nel AE: Importance of oxidative stress in the pathogenesis and treatment of asthma. Curr Opin Allergy Clin Immunol 2008;8:49-56.

4 Cho HY, Kleeberger SR: Nrf2 protects against airway disorders. Toxicol Appl Pharmacol 2010;244:43-56.

5 Li N, Nel AE: Role of the Nrf2-mediated signaling pathway as a negative regulator of inflammation: implications for the impact of particulate pollutants on asthma. Antioxid Redox Signal 2006;8:88-98.

6 Li YJ, Takizawa H, Kawada T: Role of oxidative stresses induced by diesel exhaust particles in airway inflammation, allergy and asthma: their potential as a target of chemoprevention. Inflamm Allergy Drug Targets 2010; 9:300-305.

7 Araujo JA, Barajas B, Kleinman M, Wang X, Bennett BJ, Gong KW, Navab M, Harkema J, Sioutas C, Lusis AJ, Nel AE: Ambient particulate pollutants in the ultrafine range promote early atherosclerosis and systemic oxidative stress. Circ Res 2008;102:589-596.
-8 Li N, Alam J, Venkatesan MI, Eiguren-Fernandez A, Schmitz D, Di Stefano E, Slaughter $\mathrm{N}$, Killeen E, Wang X, Huang A, Wang $M$, Miguel AH, Cho A, Sioutas C, Nel AE: Nrf2 is a key transcription factor that regulates antioxidant defense in macrophages and epithelial cells: protecting against the proinflammatory and oxidizing effects of diesel exhaust chemicals. J Immunol 2004;173:3467-3481.

-9 Reddy NM, Potteti HR, Mariani TJ, Biswal S, Reddy SP: Conditional deletion of Nrf2 in airway epithelium exacerbates acute lung injury and impairs the resolution of inflammation. Am J Respir Cell Mol Biol 2011;45:11611168.

10 Li YJ, Takizawa H, Azuma A, Kohyama T, Yamauchi Y, Takahashi S, Yamamoto M, Kawada T, Kudoh S, Sugawara I: Disruption of Nrf2 enhances susceptibility to airway inflammatory responses induced by low-dose diesel exhaust particles in mice. Clin Immunol 2008; 128:366-373.

-11 Li YJ, Takizawa H, Azuma A, Kohyama T, Yamauchi Y, Takahashi S, Yamamoto M, Kawada T, Kudoh S, Sugawara I: Nrf2 is closely related to allergic airway inflammatory responses induced by low-dose diesel exhaust particles in mice. Clin Immunol 2010;137: 234-241.

12 Kikuchi N, Ishii Y, Morishima Y, Yageta Y, Haraguchi N, Itoh K, Yamamoto M, Hizawa $\mathrm{N}$ : Nrf2 protects against pulmonary fibrosis by regulating the lung oxidant level and Th1/ Th2 balance. Respir Res 2010;11:31.
-13 Braun A, Bewersdorff M, Lintelmann J, Matuschek G, Jakob T, Gottlicher M, Schober W, Buters JT, Behrendt H, Mempel M: Differential impact of diesel particle composition on pro-allergic dendritic cell function. Toxicol Sci 2010;113:85-94.

-14 Inoue K, Koike E, Takano H, Yanagisawa R, Ichinose T, Yoshikawa T: Effects of diesel exhaust particles on antigen-presenting cells and antigen-specific Th immunity in mice. Exp Biol Med (Maywood) 2009;234:200-209.

15 Rangasamy T, Williams MA, Bauer S, Trush MA, Emo J, Georas SN, Biswal S: Nuclear erythroid 2 p 45 -related factor 2 inhibits the maturation of murine dendritic cells by ragweed extract. Am J Respir Cell Mol Biol 2010;43: 276-285.

16 Williams MA, Rangasamy T, Bauer SM, Killedar S, Karp M, Kensler TW, Yamamoto M, Breysse P, Biswal S, Georas SN: Disruption of the transcription factor Nrf2 promotes prooxidative dendritic cells that stimulate Th2like immunoresponsiveness upon activation by ambient particulate matter. J Immunol 2008;181:4545-4559.

-17 Bezemer GF, Bauer SM, Oberdorster G, Breysse PN, Pieters RH, Georas SN, Williams MA: Activation of pulmonary dendritic cells and Th2-type inflammatory responses on instillation of engineered, environmental diesel emission source or ambient air pollutant particles in vivo. J Innate Immun 2011;3:150166. 
18 Kim HJ, Barajas B, Wang M, Nel AE: Nrf2 activation by sulforaphane restores the agerelated decrease of $\mathrm{t}_{\mathrm{H}} 1$ immunity: role of dendritic cells. J Allergy Clin Immunol 2008;121: 1255-1261.

19 Li N, Wang M, Bramble LA, Schmitz DA, Schauer JJ, Sioutas C, Harkema JR, Nel AE: The adjuvant effect of ambient particulate matter is closely reflected by the particulate oxidant potential. Environ Health Perspect 2009; 117:1116-1123.

20 Hao M, Comier S, Wang M, Lee JJ, Nel A: Diesel exhaust particles exert acute effects on airway inflammation and function in murine allergen provocation models. J Allergy Clin Immunol 2003;112:905-914.

-21 Kim HJ, Barajas B, Chan RC, Nel AE: Glutathione depletion inhibits dendritic cell maturation and delayed-type hypersensitivity: implications for systemic disease and immunosenescence. J Allergy Clin Immunol 2007; 119:1225-1233.

22 Kuipers H, Soullie T, Hammad H, Willart M, Kool M, Hijdra D, Hoogsteden HC, Lambrecht BN: Sensitization by intratracheally injected dendritic cells is independent of antigen presentation by host antigen-presenting cells. J Leukoc Biol 2009;85:64-70.

23 Blake DJ, Singh A, Kombairaju P, Malhotra D, Mariani TJ, Tuder RM, Gabrielson E, Biswal S: Deletion of Keap1 in the lung attenuates acute cigarette smoke-induced oxidative stress and inflammation. Am J Respir Cell Mol Biol 2010;42:524-536.

-24 Rangasamy T, Guo J, Mitzner WA, Roman J, Singh A, Fryer AD, Yamamoto M, Kensler TW, Tuder RM, Georas SN, Biswal S: Disruption of Nrf2 enhances susceptibility to severe airway inflammation and asthma in mice. J Exp Med 2005;202:47-59.
Reddy NM, Kleeberger SR, Kensler TW, Yamamoto M, Hassoun PM, Reddy SP: Disruption of Nrf2 impairs the resolution of hyperoxia-induced acute lung injury and inflammation in mice. J Immunol 2009;182: 7264-7271.

26 Braback L, Forsberg B: Does traffic exhaust contribute to the development of asthma and allergic sensitization in children: findings from recent cohort studies. Environ Health 2009;8:17.

27 Castro-Giner F, Kunzli N, Jacquemin B, Forsberg B, de Cid R, Sunyer J, Jarvis D, Briggs D, Vienneau D, Norback D, Gonzalez JR, Guerra S, Janson C, Anto JM, Wjst M, Heinrich J, Estivill X, Kogevinas M: Traffic-related air pollution, oxidative stress genes, and asthma (ECHRS). Environ Health Perspect 2009;117: 1919-1924.

28 Piacentini S, Polimanti R, Moscatelli B, Re MA, Fuciarelli R, Manfellotto D, Fuciarelli M: Glutathione S-transferase gene polymorphisms and air pollution as interactive risk factors for asthma in a multicentre Italian field study: a preliminary study. Ann Hum Biol 2010;37:427-439.

29 Yang IA, Fong KM, Zimmerman PV, Holgate ST, Holloway JW: Genetic susceptibility to the respiratory effects of air pollution. Thorax 2008;63:555-563.

30 O'Garra A, Murphy KM: From IL-10 to IL-12: how pathogens and their products stimulate apcs to induce $t_{\mathrm{H}} 1$ development. Nat Immunol 2009;10:929-932.

31 Bilenki L, Gao X, Wang S, Yang J, Fan Y, Han X, Qiu H, Yang X: Dendritic cells from mycobacteria-infected mice inhibits established allergic airway inflammatory responses to ragweed via IL-10- and IL-12-secreting mechanisms. J Immunol 2010;184:7288-7296.

-32 Ohtani T, NakagawaS, Kurosawa M, Mizuashi M, Ozawa M, Aiba S: Cellular basis of the role of diesel exhaust particles in inducing Th2dominant response. J Immunol 2005; 174: 2412-2419.
33 Porter M, Karp M, Killedar S, Bauer SM, Guo J, Williams D, Breysse P, Georas SN, Williams MA: Diesel-enriched particulate matter functionally activates human dendritic cells. Am J Respir Cell Mol Biol 2007;37:706-719.

-34 Doganci A, Sauer K, Karwot R, Finotto S: Pathological role of IL- 6 in the experimental allergic bronchial asthma in mice. Clin Rev Allergy Immunol 2005;28:257-270.

35 Andre S, Tough DF, Lacroix-Desmazes S, Kaveri SV, Bayry J: Surveillance of antigen-presenting cells by CD4+ CD25+ regulatory $\mathrm{T}$ cells in autoimmunity: immunopathogenesis and therapeutic implications. Am J Pathol 2009;174:1575-1587.

36 Diehl S, Chow CW, Weiss L, Palmetshofer A, Twardzik T, Rounds L, Serfling E, Davis RJ, Anguita J, Rincon M: Induction of NFATc2 expression by interleukin 6 promotes T helper type 2 differentiation. J Exp Med 2002;196: 39-49.

37 Chow JY, Wong CK, Cheung PF, Lam CW Intracellular signaling mechanisms regulating the activation of human eosinophils by the novel Th2 cytokine IL-33: implications for allergic inflammation. Cell Mol Immunol 2010; 7:26-34.

38 Cassel SL, Joly S, Sutterwala FS: The NLRP3 inflammasome: a sensor of immune danger signals. Semin Immunol 2009;21:194-198.

39 Provoost S, Maes T, Pauwels NS, Vanden Berghe $\mathrm{T}$, Vandenabeele $\mathrm{P}$, Lambrecht $\mathrm{BN}$, Joos GF, Tournoy KG: NLRP3/caspase-1-independent IL- $1 \beta$ production mediates diesel exhaust particle-induced pulmonary inflammation. J Immunol 2011;187:3331-3337.

40 Barlow JL, Bellosi A, Hardman CS, Drynan LF, Wong SH, Cruickshank JP, McKenzie $\mathrm{AN}$ : Innate IL-13-producing nuocytes arise during allergic lung inflammation and contribute to airways hyperreactivity. J Allergy Clin Immunol 2012;129:191-198. 